
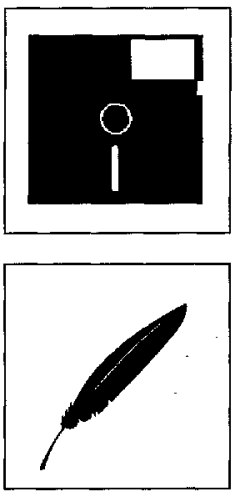

Das Hauptmenü

\title{
Das HGB-Lernprogramm Handelsrecht ${ }^{1}$
}

\section{Götz Knoop}

Computer sind als didaktische Hilfsmittel auch in der Juristenausbildung nichts Neues mehr. Bereits seit Anfang der achtziger Jahre wird an deutschen Hochschulen am didaktischen Einsatz des PC im Rahmen von Forschungsprojekten und Seminararbeiten gearbeitet. ${ }^{2}$ Trotzdem ist das Angebot an tutoriellen Lernsystemen, d.h. Programmen, die den juristischen Lernstoff vorgeben und deren hauptsächlicher und unmittelbarer Zweck in der Wissenspräsentation und somit in der Darstellung von Begriffen und Zusammenhängen des Fachgebietes liegt, bislang nicht sehr reichhaltig. ${ }^{3}$ Insbesondere solche tutoriellen Systeme, die einzelne Teilbereiche vollständig abdecken, sind Mangelware. ${ }^{4}$ Zudem schien die Entwicklung tutorieller juristischer Lernsysteme auf kommerzieller Ebene ins Stokken geraten zu sein. ${ }^{5}$ Im Juli 1994 unternahmen Studenten, die zu diesem Zweck den FGL-Verlag gründeten, einen neuen Vorstoß, indem sie das HGB-Lernprogramm Handelsrecht herausgaben. Mit diesem Lernprogramm will der Verlag nicht nur Jurastudenten ansprechen. Vielmehr soll das Programm für Kaufleute, Studenten der Volks- und Betriebswirtschaftslehre, Juristen und Jurastudenten geeignet sein.

\section{Die Installation}

Das Programm wird auf einer 3 1/2 Zoll Diskette geliefert, auf der sich eine komprimierte Datei befindet. Diese Datei entpackt sich selbst, nachdem sie auf die Platte kopiert und aufgerufen wurde. Die Notwendigkeit der Entkomprimierung hat im Handbuch leider keinen Niederschlag gefunden, so daß Anwender ohne computertechnische Erfahrung hier bereits vor die erste Hürde gestellt werden. Vermißt wird bei der vorliegenden Version auch eine Installationsroutine, die ein Icon unter Windows einrichtet. Dies muß der Anwender per Hand vornehmen, was aber einem geübten Windows-Anwender keinerlei Probleme bereiten dürfte. Außerdem soll dieser Schönheitsfehler

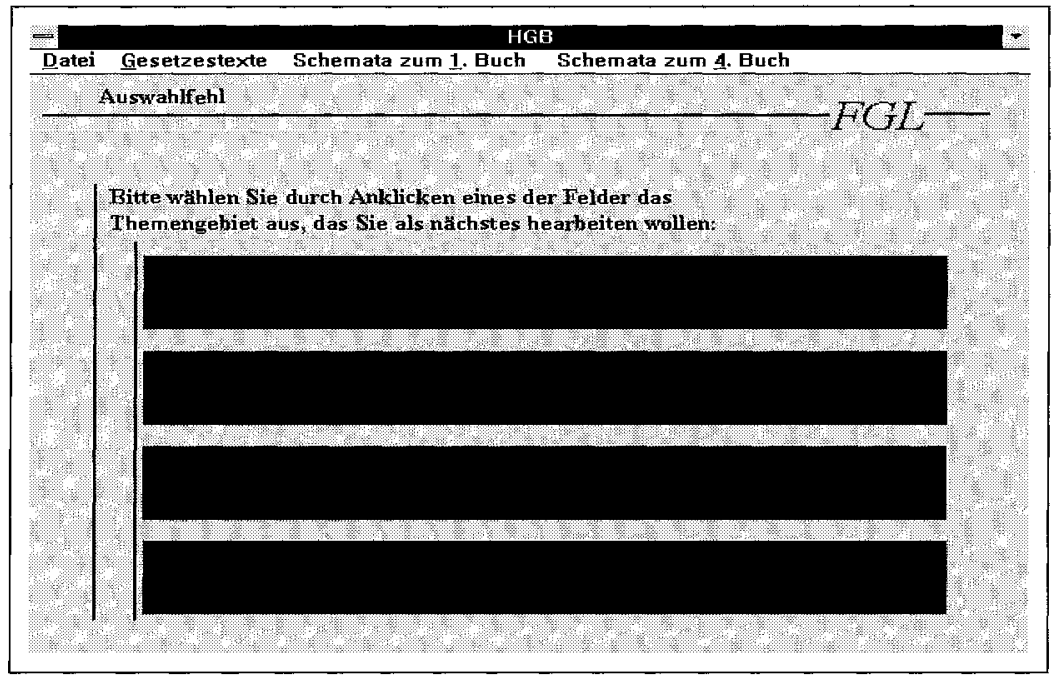

nach Auskunft der Autorin bereits beseitigt sein.

\section{Die Systematik}

Das Programm ist in vier Abschnitte eingeteilt. Im ersten Abschnitt wird eine Einführung in die Bearbeitung juristischer Fälle gegeben, was insbesondere angesichts der weitgefaßten Zielgruppe recht sinnvoll erscheint. Der zweite und dritte Abschnitt sind dem ersten und vierten Buch des HGB gewidmet, im vierten Abschnitt stehen drei Übungsklausuren zur Auswahl (vgl. Abb. 1).

\section{Wissenspräsentation}

Während der erste Abschnitt Fallösungstechnik und Aufbaufragen klären soll und der vierte Abschnitt dem Training der Wissensanwendung dient, geht es im zweiten und dritten Abschnitt um die eigentliche Wissenspräsentation. Diese beiden Abschnitte sind wiederum in Unterabschnitte gegliedert, die sich mit einzelnen Themenfeldern wie Kaufmannseigenschaft oder Prokura und Handlungsvollmacht auseinandersetzen.

\section{Gesetzestext als Ausgangspunkt}

Ausgangspunkt dieser Wissenspräsentation ist im Programm immer der Gesetzestext. Dem Anwender werden die einzelnen Paragraphen des jeweiligen Un-

\footnotetext{
Dem Rezensenten lag die im September 1994 verfügbare erste Version des Programms vor.

Andreas Günther in jur-pc 90, 759

vgl. Andreas Günther aaO.

4 Von Alpmann \& Schmidt sind zwei Programme, eines zum allgemeinen Teil des BGB und eines zum Recht der beweglichen Sachen, erschienen.

5 Alpmann \& Schmidt hat sein letztes Programm Ende 1991 auf den Markt gebracht und seitdem nicht überarbeitet. Wie jedoch vom Verlag zu erfahren war, erscheint Anfang 1995 ein Programm zum Verwaltungsrecht AT.
} 
terabschnittes auf dem Bildschirm angezeigt (vgl. Abb. 2). Durch Doppelklick auf dem jeweiligen Paragraphen kann sich der Anwender den Gesetzestext anzeigen lassen, wobei Kommentierungen $\mathrm{zu}$ den einzelnen Tatbestandsmerkmalen im Wege der Hypertexttechnik ${ }^{6}$ realisiert sind. Neben dieser Angabe des Gesetzestextes hat der Anwender die Möglichkeit, sich Schemata und Übersichten mit Hilfe eines Schalters beziehungsweise PullDown-Menüs auf den Bildschirm zu rufen (vgl. Abb. 2).

Bei der Wissenspräsentation ist begrüßenswert, daß eng am Gesetzestext gearbeitet wird. Von einem Tatbestandsmerkmal ausgehend wird erläutert, wie sich das jeweilige Merkmal in die Rechtsgebiete ein- und unterordnet, beziehungsweise wie die entsprechende Definition lautet. Unterstützt wird dies durch o. g. Schemata und Übersichten.

Eine andere Methode der Wissenspräsentation wäre es gewesen, von Übersichten auszugehen und anhand dieser Übersichten die Systematik der Regelungen zu erläutern.

Welche Methode hier die bessere ist, werden die Lernerfolge zeigen.

\section{Nachteile}

Ein großer Nachteil der Wissenspräsentation ist beim Lernprogramm Handelsrecht der Umstand, daß das Wissen grundsätzlich in abstrakter Form dargeboten wird. Es werden kaum Beispiele geboten, die die konkrete Anwendung am Sachverhalt zeigen würden.

Ein weiterer Nachteil des Präsentationsteils ist, daß sich das Programm nicht auf den Level des jeweiligen Anwenders einstellen kann. Eine Unterteilung in verschiedene Lernstufen, wie es bei eTutor ${ }^{7}$ vorgenommen wurde, ist im Lernprogramm Handelsrecht nicht zu finden. Wenn die Autorin auf dem Schutzumschlag der Programmdiskette individuelle Lerninhalte verspricht, muß sie wohl etwas anderes gemeint haben als eine solche Unterteilung.

\section{Abfragemechanismen}

Beim Anklicken des entsprechenden Schalters, spätestens aber automatisch am Ende eines jeden Unterabschnittes, erfolgt eine Wis-

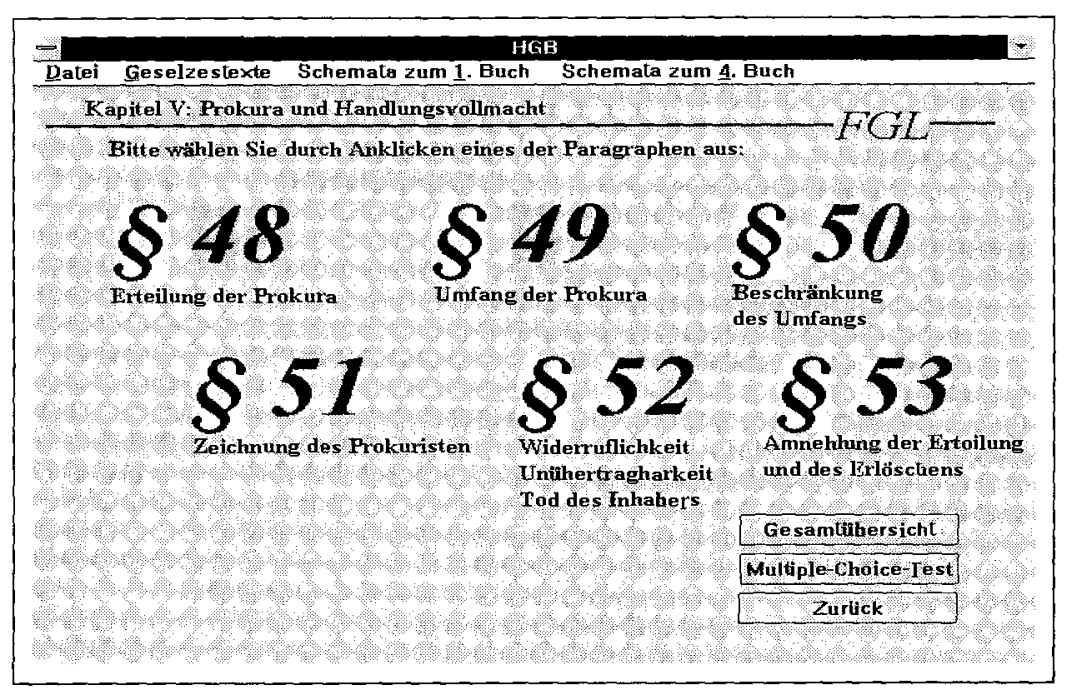

sensüberprüfung in Form eines Tests. Dieser Test setzt sich zum überwiegenden Teil aus Multiple-Choice-Fragen zusammen. Daneben existieren Fragen mit Freitext- und Lückentexteingaben. Am Ende eines Testes wird eine Auswertung vorgenommen. Hier wird die Anzahl der richtig beantworteten Fragen der Gesamtzahl der gestellten Fragen gegenübergestellt. ${ }^{8}$ Teilweise werden Fragen dieses Testes schon während der Durcharbeitung der Wissenspräsentation eingestreut.

\section{Problem: Schreibfehler}

Der Nachteil der Wissensüberprüfung durch Freitexteingabe ist, daß Schreibfehler und ähnliches kaum abgefangen werden können. So verhält es sich auch in diesem Programm. Zum Beispiel akzeptiert das Programm bei der Frage in Abb. 3 (auf der folgenden Seite) die - orthographisch nicht ganz korrekte - Antwort in fremden Namen nicht, sondern gibt sich erst mit in fremdem Namen zufrieden. Mit einer Rüge bei der ersten Antwort ruft das System beim Anwender zunächst Verwirrung hervor, die erst durch die Anzeige der Musterantwort geklärt wird.

Bedauernswert ist außerdem, daß im Lernprogramm Handelsrecht keinerlei andere Abfragemechanismen zur Anwendung gekommen sind. Insbesondere bei der Überprüfung von Definitionskenntnissen wäre es möglich gewesen, dies anhand von Fällen durchzuführen, wobei der Anwender angeben müßte, ob der Sachverhalt unter die Definition subsumiert werden kann und an welchem Definitionsmerkmal es gegebenenfalls scheitert. ${ }^{9}$ Bei dieser Methode würde nicht nur der
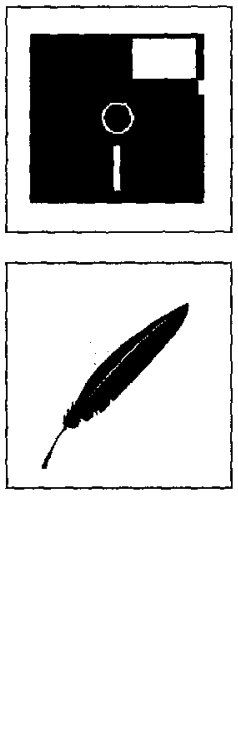

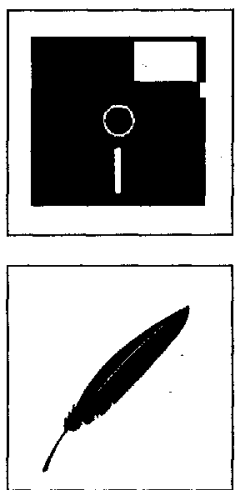

Wortlaut einer Definition überprüft, sondern auch die Fähigkeit ihrer Anwendung.

\section{Der Inhalt}

Der erste Abschnitt soll der Einführung in die Bearbeitung juristischer Fälle dienen. In diesem $\mathrm{Ab}$ schnitt wird eindringlich darauf hingewiesen, daß die Formalien, der Gutachtenstil und auch die Subsumtionstechnik streng einzuhalten sind. Dieser Hinweis ist sicherlich richtig und wichtig. Aber eine auch nur ausreichende Angabe dessen, was die Formalien, den Gutachtenstil und die Subsumtionstechnik ausmacht, wird leider nicht gegeben. Lediglich hinsichtlich des Gutachtenstils wird erwähnt, daß dieser sich aus Obersatz, Voraussetzungen, Subsumtion und Ergebnis zusammensetzt. Eine detailliertere Erläuterung ${ }^{10}$ wird nicht gegeben. Auch erscheint keinerlei Hinweis auf weiterführende Literatur zur juristischen Systematik. ${ }^{11}$

\section{Wissenspräsentation}

Was den Präsentationsteil des zweiten und dritten Kapitels anbelangt, ist zunächst festzuhalten, daß das Programm nach Auskunft eines Verlagsmitarbeiters den Anforderungen der Nebenfachprüfung im ersten Examen genügen soll. Insofern ist festzustellen, daß alle examensrelevanten Themengebiete im Programm angesprochen werden. Teilweise mangelt es aber an einer vollständigen und genauen Darstellung. So wird zum Beispiel die $\mathrm{Ab}$ grenzung zwischen Zivil- und Handelsmakler als Kommentierung zum Tatbestandsmerkmal gewerbsmäßig des $\$ 93 \mathrm{HGB}$ vorgenommen (vgl. Abb. 4, auf der folgenden Seite), was den Schluß zuläßt, daß das Abgrenzungskriterium das Merkmal der Gewerbsmäßigkeit sei. Bezeichnenderweise hat die Autorin zur Figur des kaufmännischen Zivilmaklers keinerlei Stellung mehr bezogen. Daß diese Unterscheidung falsch ist, bestätigt nicht nur Palandt ${ }^{12}$, sondern dürfte all denen, die das Handelsrecht kennen, bekannt sein. Der typische Anwender eines Lemprogramms kennt das jeweilige Gebiet jedoch gerade (noch) nicht. Daher sind solche Fehler schwerwiegend. Um so gravierender ist es, daß solche Fehler mit Unvollständigkeiten einhergehen. So wird die Untersuchungs- und Rügepflicht beim Handelskauf im Programm zwar angesprochen, jedoch wurden Abs. 2 und 3 des $\$ 377$ in keiner Weise kommen-

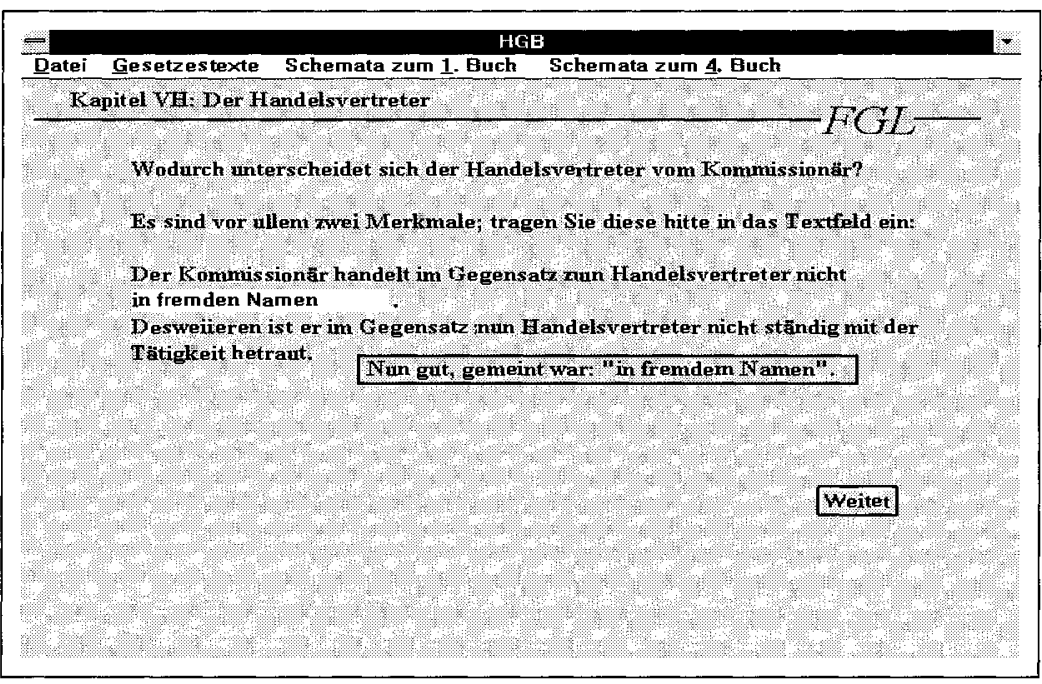

tiert. Die - durchaus examensrelevante - Unterscheidung zwischen versteckten und offenkundigen Mängeln einerseits und dem Umfang der Untersuchungspflicht andererseits wurde somit im Programm nicht erwähnt.

\section{Abfragemechanismen}

Eine Betrachtung der Abfragemechanismen offenbart leider, daß die Fehler und die Unvollständigkeit der Wissenspräsentation keine einmaligen Ausrutscher sind.

So hat sich das falsche Unterscheidungskriterium zwischen Handelsund Zivilmakler auch in einer Frage niedergeschlagen (vgl. Abb. 5, auf der folgenden Seite).

Folgenschwer ist hier besonders, daß nicht nur bei der Abfrage von Fakten, sondern auch bei Verständnisfragen Fehler gemacht wurden. So soll auf die Frage nach den Personen, die ein Handelsgewerbe betreiben (vgl. Abb. 6) die Antwort Alle richtig sein. Hier hat die Autorin entweder die Begriffe Grundhandelsgewerbe und $\mathrm{Ge}$ werbe durcheinander gebracht, oder es handelt sich lediglich um einen programmiertechnischen Fehler. Beides ist für den - typischerweise im Handelsrecht noch unkundigen - Anwender nicht ohne weiteres erkennbar. Darüber hinaus sind die Musterantworten teilweise schlecht formuliert. Wenn auf eine Multiple-ChoiceFrage von zwei möglichen Antworten die eine lautet: "... eine lausige Vorschrift", und somit offensichtlich nicht ernsthaft in Betracht kommt, leuchtet der Lernwert dieser Frage nicht mehr ein.

\section{Schlußbetrachtung}

Sehr erfreulich ist zunächst die Tatsache, daß sich auf dem Sek-

10 vgl. etwa Schmalz, Methodenlehre, 1. und 7. Teil

11 aktuellstes Buch: Schmalz, Methodenlehre, 3. Aufl., Münster, 1993

12 Palandt, BGB, 51. Aufl., Einf vor $\$ 652$ Rz. 2 
tor der kommerziellen tutoriellen Lernprogramme seit fast drei Jahren wieder etwas bewegt hat. Erfreulich ist auch der Mut der Autorin, auf dem Bereich der Wissensvermittlung einen anderen Weg einzuschlagen. Die Vorgehensweise, vom Gesetzestext ausgehend die einzelnen Tatbestandsmerkmale hinsichtlich ih- rer Bedeutung und Ein- bzw. Unterordnung in die Rechtsgebiete zu erläutern, ist zu begrüßen. Sie fördert die Fähigkeit, sich mit Hilfe des Gesetzestextes und nur der liegt im Examen vor - Problembereiche zu erschließen und Lösungen zu finden. $\mathrm{Ob}$ sich diese Art der Wissensvermittlung in tutoriellen
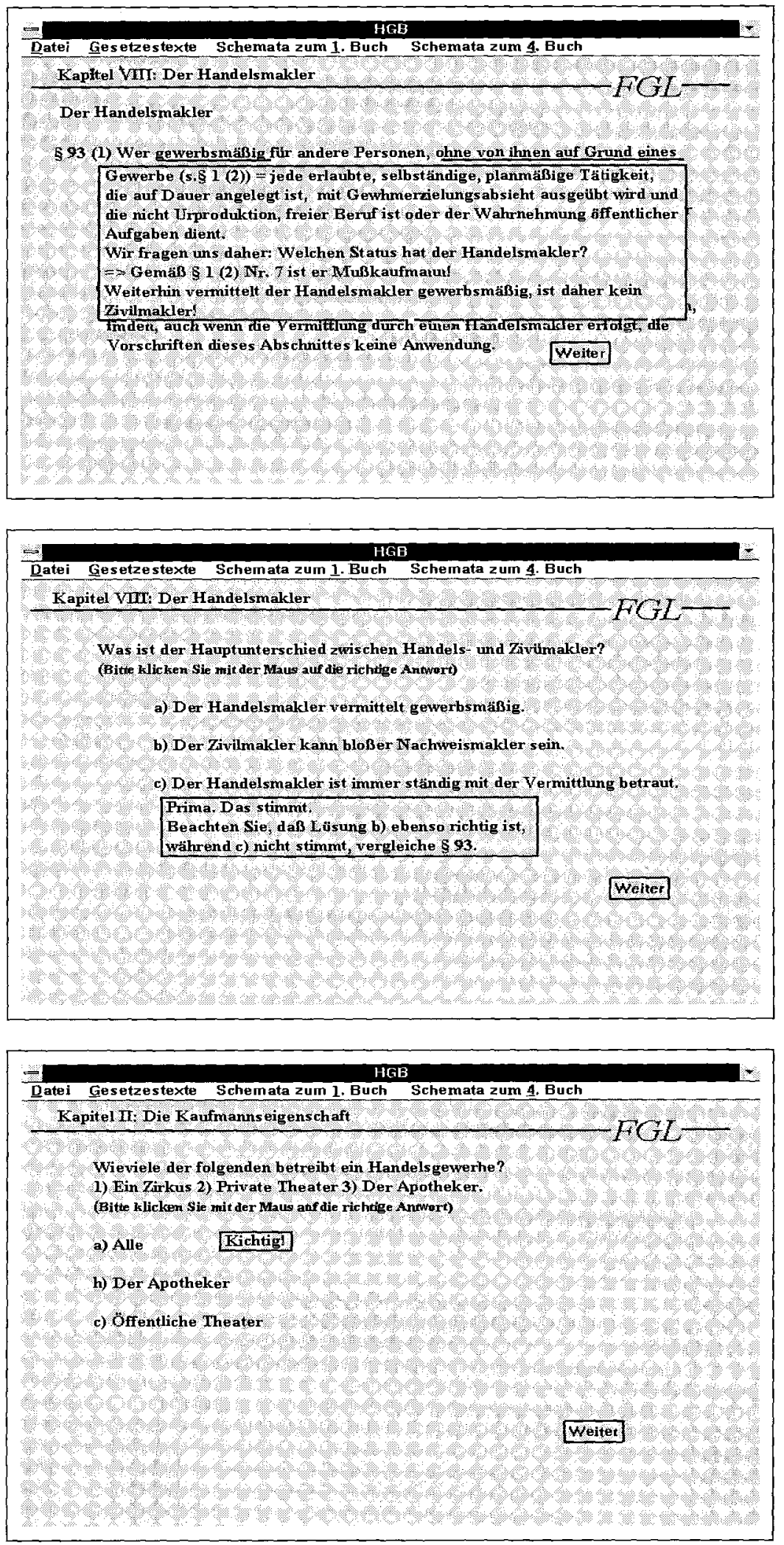

Lernprogrammen durchsetzt, bleibt abzuwarten.

Dieser positive Eindruck wird jedoch durch die vorhandenen Fehler stark abgeschwächt. So mögen zwar $95 \%$ der Wissenpräsentation fehlerfrei sein, jedoch zerstören die $5 \%$ Fehlerhaftigkeit die Vertrauensgrundlage des Lernenden in das gesamte Lernprogramm. Der noch unkundige Anwender weiß eben nicht, ob ein bestimmter Punkt zu den $95 \%$ oder zu den $5 \%$ zählt. Er wird sich daher ein Lehrbuch nehmen und nachlesen müssen. Wenn der Anwender dann das Themengebiet im Lehrbuch nacharbeitet, wird er die vorherige Anschaffung des Lernprogramms möglicherweise als überflüssig empfinden.

Unbefriedigend ist leider der $\mathrm{Be}$ reich der Abfragemechanismen ausgefallen. Nicht nur das denkbare Abfragetechniken nicht genutzt werden, sondern auch die Fehler - insbesondere die bei Verständnisfragen - schlagen negativ zu Buche. Diese Fehler haben beim Rezensenten den Eindruck unprofessioneller Programmerstellung erweckt. Zwar wurde das Programm Handelsrecht von Studenten erstellt, aber es nimmt für sich höchste Professionalität in Bezug auf die Vorbereitung zur ersten juristischen Staatsprüfung in Anspruch. Diesen selbst gesetzten Anspruch kann es alleine nicht halten. Es ist allenfalls als Ergänzung zu den üblichen Lernmedien (wie Kommentare und Lehrbücher) denkbar.

\section{Bezugsquelle:}

Das HGB-Lernprogramm 'Handelsrecht' ist zum Preis von DM 49,80 über den Buchhandel zu beziehen oder kann direkt beim FGL-Verlag, Scheibenstr. 84, 48153 Münster, bestellt werden.

Technische Voraussetzungen:

- PC ab 386

- Windows ab Version 3.0

- ca. 3 MB Festplattenspeicher

- 3 1/2 Diskettenlaufwerk (5 1/4 Version kann bestellt werden)
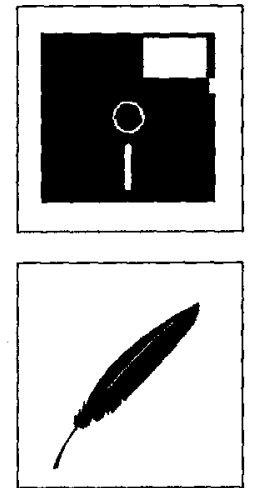

Abb. 4

Abgrenzung zwischen Zivil-und Handelsmakler

Abb. 5

Falsches Unterscheidungskriterium zwischen Han dels- und Zivilmakler hat sich auch in einer Frage niedergeschlagen.

$A b b .6$ Falsche Verständnisfrage 